\title{
Impact of Physical Deformation on Electrical Performance of Paper-based Sensors
}

\author{
Joanna M. Nassar, and Muhammad M. Hussain, Senior Member, IEEE
}

40

Abstract-We report on investigation of the mechanica 1 properties of paper electronics (printed and made out of paper) 42 One key objective of such paper electronics is to achieve ultra 43 flexibility. Therefore, it is important to understand electrical 4 functionality and reliability of paper electronics under various physical (mechanical) deformation. Here we show, the generat 5 mechanical properties of the cellulose paper used and it $\$ 6$ electrical behavior under applied strain, tackling the main effect $\$ 7$ that need to be identified when building paper based systems 48 from product performance and stability perspective. An overviey 9 of the stress-strain behavior of silver ink on paper is discussed and then we tackle a more specific analysis of the performance variations of paper sensors made with recyclable household 1 materials when exposed to various mechanical conditions of 2 tensile and compressive bending. This study is important fō̄3 developing stable wearable sensors for incorporation in Internet 4 of Everything (IoE) applications.

Index Terms - Non-uniform anisotropic material, Cellulose paper, Sensors, Thin-film metal.

\section{INTRODUCTION}

59

${ }_{2}^{2} \mathrm{P}$ aper is one of the most commonly used affordable flexibl 60 materials. Although it has been used for our daily use in many different ways, its usage as a substrate for electronics is an interesting concept. The main constituent of paper is woo 4 or plant fibers (or furnish [1]). The mechanical properties of paper sheets prepared in laboratories defer from commerciall 66 manufactured ones (like the Post-It ${ }^{\mathrm{TM}}$ note paper used in this work), even though the same raw materials are used Constitutive laws are generally used to analyze the mechanical 6 properties of paper, and it has been shown that temperaturg has little effect on its deformation under strain, whereas the effect of moisture is more prominent [1]. Typically, stress and strain quantities are defined for continuous materials, however, paper is only considered as a continuous material down to centimeter scale, below which the network of cellulose fibers 3 starts to dominate, and non-uniform randomness prevails. In $^{4}$ this case, only models and approximations derived from 75

This publication is based upon work supported by the King Abdullah 77 University of Science and Technology (KAUST) Office of Sponsored 8 Research (OSR) under Award No. Sensor Innovation Initiative OSR-201579 Sensors-2707 and KAUST-KFUPM Special Initiative OSR-2016-KKI-2880. 80

J. M. Nassar and M. M. Hussain are with the Integrated Disruptive Electronic Applications (IDEA) Lab and Integrated Nanotechnology La\&1 (INL), Electrical Engineering, Computer Electrical Mathematical Science an82 Engineering Division, King Abdullah University of Science and Technologg3 (KAUST), Thuwal 23955-6900, Saudi Arabia. (E-mails. Joanna.nassar@kaust.edu.sa and muhammadmustafa.hussain@kaust.edu.sa). 84 macroscopic behaviors can be chosen to represent the material's behavior in paper electronics. In fracture processes, deformations take place at very small scales close to a crack, and the behavior of the material at that scale should be determined. Many mechanical properties for ultra-thin sheets are affected by the elastic modulus of the paper, and the mechanics of paper can be extensive. Here we show, mechanical properties (under various physical deformation) of interest necessary to explain the electrical behavior of paperbased sensors for wearable electronic applications.

In a recent report, we demonstrated the fabrication of highperformance multisensory paper-based artificial skin, "Paper Skin" (Fig. 1a) for the simultaneous detection of environmental and external stimuli in real-time [2]. This platform sees a variety of applications in wearables for the future Internet of Everything (IoE) era. In this case, assessing the behavior of the sensors, and thus the system, under various mechanical bending conditions, as depicted in Fig. 1b and c, is one of the essential building blocks for the development of a robust wearable system. Accordingly, we first study the mechanics of silver ink thin film on top of a compliant flexible Post-It paper, which will provide an essential overview of system interconnects behavior and stability over a wide range of applied tensile and compressive strains. Then, we illustrate the effect of concave (Fig. 1d and e) and convex bending (Fig. 1f) of the different sensory structures and the corresponding performance change for the demonstrated paper sensors (temperature, pressure and humidity sensors) (Fig. 1a), referred to in our previous report [2]. Please note that our sensors platform is only made out of paper as the substrate, and the Kapton tape/sheet seen in Fig. 1 has been only used for support purposes and taking the various digital photos.

\section{Theory Of PAPER Stress-Strain Mechanics}

Unlike other materials, the elastic modulus $\mathrm{E}$ of ultra-thin paper is highly anisotropic and paper is approximated to be orthotropic, meaning that the stiffness properties are symmetric with respect to the $\mathrm{x}, \mathrm{y}$ and $\mathrm{z}$ axes of its plane [1]. Strictly speaking, in Hooke's law, the elastic moduli E and Poisson's ratio ' $v$ ' are applicable only in the ideal case of a perfectly linear elastic material. It is typical for any grade of paper to see a decrease in the elastic modulus by around $10 \%$ before the breaking point, even though part of the strain is irreversible [1]. Nevertheless, measured stress-strain curves of paper are approximately linear elastic within certain loading, where in general, the in-plane elastic modulus of paper increases with density $\left(\mathrm{kg} \cdot \mathrm{m}^{-3}\right)$. 

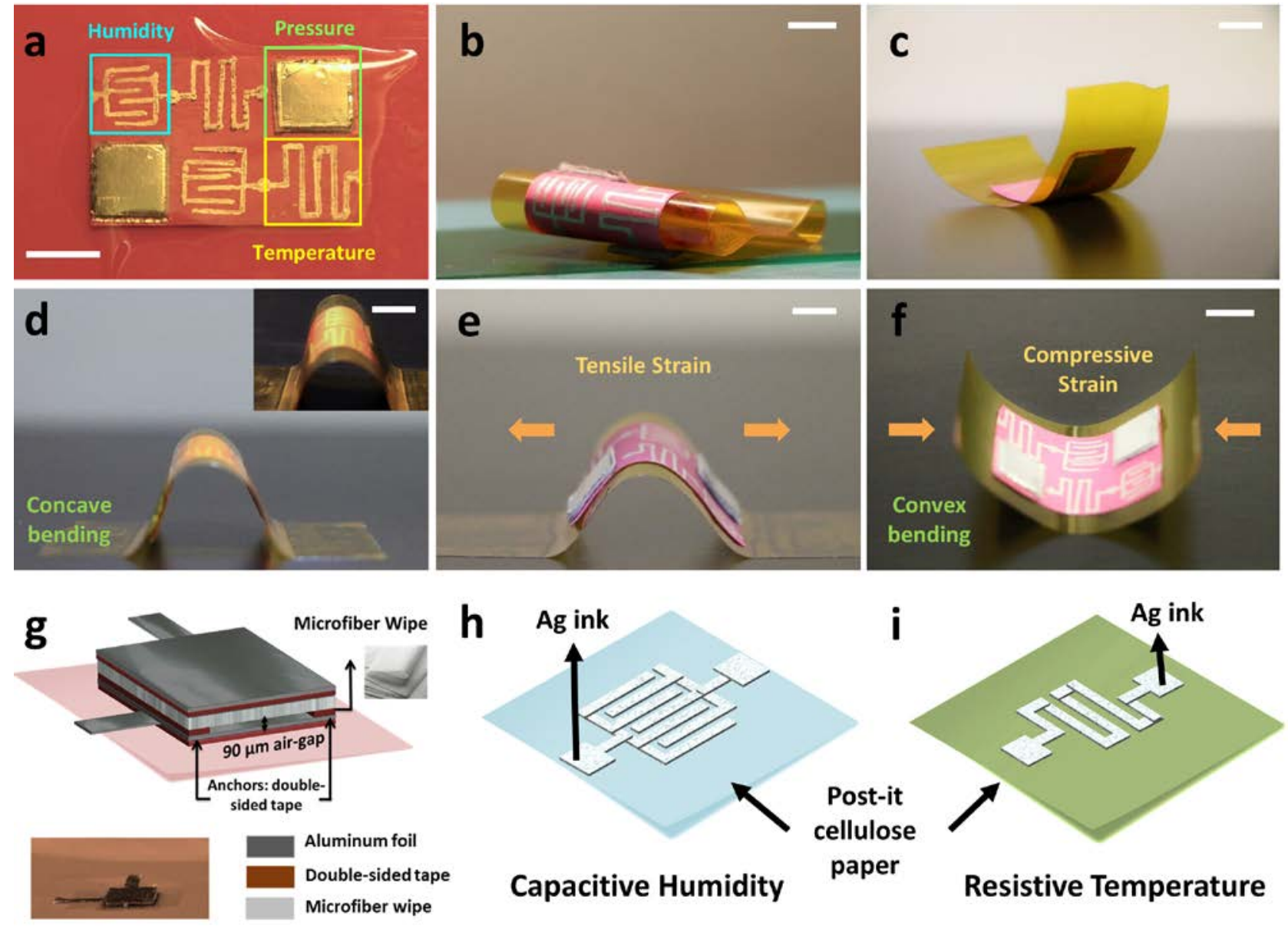

Fig. 1. (a) Multisensory paper-based artificial skin "Paper Skin”, displaying in-plane integration of pressure, temperature and humidity sensors. (b) and (c) Digital photos of Paper skin supported on a Kapton sheet tape, revealing its flexibility and robustness under flexure and bending. (d) and (e) Digital photos of the different concave bending setups of Paper Skin exerting a tensile strain on the structures, with an inset image highlighting the top view of the platform. (f) Digital photograph depicting the convex bending of the sensory platform, which translates into a compressive strain being applied. (g) Schematic illustrating the parallel-plate capacitive structure of the pressure sensor. (h) Schematic of the interdigitated capacitive structure of the humidity sensor. (i) Schematic of the resistive structure of the temperature sensor.

Moreover, since we are fabricating paper based humiditz4 sensors, it is to be noted that water acts as a softener of pape2,5 thus the elastic modulus of paper depends on the moistura6 content, where higher relative humidity levels (\% RH) in tha7 atmosphere would translate into a decrease in the elasti28 modulus of paper, approaching zero as the bonding between 9 the fibers opens. This process highlights the reversibility a 0 the papermaking process, hence making it a recyclabla1 material [1]. In this manner, the tensile stiffness (which is th 32 elastic modulus multiplied by paper thickness) decrease33 drastically with moisture content. This softening effect make34 paper both visco-elastic and visco-plastic, meaning that the5 slope of the measured stress-strain curves (i.e. the apparen 6 modulus) increases significantly as strain rate increases 37 However, when relative humidity levels are equal or higheß8 than $50 \% \mathrm{RH}$, the elastic modulus of paper is governed by the interactions between fibers mediated by liquid wateß9 Therefore, any stress created through deformations and 0 bending would rapidly relax to zero, improving the durability1 of deposited films and devices built on paper, and the breaking 2 strain of the paper becomes higher at higher relative humidity 43
Under in-plane tensile loading, the elastic modulus of paper changes a little before the peak stress, suggesting that the network of microscopic fibers undergoes permanent plastic deformations that do not weaken the elastic stiffness of the fibers [1]. And after the peak stress, the elastic modulus decreases, allowing for stable device performance under tensile bending conditions, where the large ductility of fibers mainly comes from the length of the fibers. For in-plane compressive loading, the mechanics are a bit more complicated on the thin planar paper material. However, buckling needs to be prevented when the sample is bent, and still paper would fail under compressive stress faster than under tensile stress, mainly due to the behavior of pores where pore volume closes and the apparent stiffness of the paper increases rapidly towards infinity [1].

\section{Bending Mechanics OF ThIN FILM ON PAPER}

It is important to note that wearable electronics will be subjected to anomalous physical deformation based on user habits and activities. Such mechanical anomalies will now be serving as independent variable in the electrical reliability of 
1 the wearable electronics. Hence, for applications of wearablis7

2 sensors it is important to understand how the different set o\$8 3 materials behave under bending conditions. For our reporte89 4 paper-based sensors, we use silver ink pen (Circuit Scribe ${ }^{\mathrm{TM}} \mathrm{g} 0$ 5 to draw our sensors structures and metal interconnects. Thus 1 6 it is crucial to understand the behavior and variations in th62 7 electrical and topographical properties of the silver metal filr63 8 under different strain levels. This is important as the sensorø4 9 are implemented in a wearable application, and the limitation65 10 of metal delamination or discontinuity need to be studie66

beforehand. Therefore, in this section we test th 67 topographical changes in the microstructure of the silver in 68 film when exposed to tensile strain down to bending radius 189 $=2 \mathrm{~mm}$. When the platform is concave bent, the metal layer0 (i.e. silver ink film) undergoes slight strain, leading to a linea $\mathbf{r} 1$ increase in resistance: as strain " $\varepsilon$ " increases, resistance "r72 increases following $\Delta \mathrm{r} / \mathrm{r}_{0}=\mathrm{GF} \times \varepsilon$, where GF is the gage 3 factor or strain sensitivity and " $\mathrm{r}_{0}$ " is the initial resistanc $\mathbb{4} 4$ under no strain. Details about the effect of different strain levels on the sensors characteristics will be thoroughly $y_{6}$ discussed in the next section.

The Circuit Scribe silver ink used in this work is mainly 77 composed of silver and water [3], where silver is ten times denser than water. However, in this work, the silver ink has been annealed at $\mathrm{T}=100{ }^{\circ} \mathrm{C}$, evaporating any water content in our metal film. The silver ink coats the fibrous paper surface in a conformal manner, leading to good adhesion and no observed delamination between the metal film and the paper 84 upon bending. Since the substrate used is flexible fibrous 85 paper, the silver layers see minimal strain. Thus no major 5 crack formation or delamination is observed during the first 6 bending cycles. Mechanics or stress-strain behavior of 87 cellulose paper under tensile loading (bending direction in this work), exhibits both linear and nonlinear behaviors, where 89 time-dependence and stress relaxation of paper plays a big role ${ }^{90}$ [1]. During a tensile in-plane stress-strain measurement of paper, the elastic modulus changes very little even though part of the strain is irreversible or plastic [1]. This is typical of almost all paper grades, where the elastic modulus decreases 94 by a maximum of $10 \%$ before reaching the breaking point. 95 Post-It ${ }^{\mathrm{TM}}$ used in this work is classified under the ductile grad $\mathrm{e}^{6}$ of paper, where a modest increase in elastic modulus can be ${ }^{7}$ seen. Elastic modulus of paper changes only little before peak 8 stress is achieved, and after peak stress the elastic modulus 9 decreases. This shows that when the microscopic cellulose 100 network of paper undergoes a permanent plastic deformation, 1 this does not weaken the elastic stiffness of the fibers [1]. 1 Q2 our case, very minimal stress is applied, thus elastic modul 163 is assumed to be stable. As an approximation, studies showę 4 that typical cellulose fiber based papers have an anisotropi 65 elastic modulus of around $5 \mathrm{GPa}$, which is usually ten time日6 6 lower than the standalone elastic modulus of cellulose materimb 7 [4]. This Young's modulus will generally depend on th98 random orientation of the fibers, the porosity of paper, and thig fiber properties. For in-plane tensile stress-strain curves, the typical breaking strain point of paper ranges from $1 \%$ to $5 \%$ Pf 1

applied strain. However, moisture content plays a big role in softening the paper, where a higher relative humidity (RH \%) is translated to a decrease in the elastic modulus of paper as the bonding between fibers opens [1]. Thus, the breaking strain point increases with increased moisture content. In contrast, for in-plane compression mode, stress-strain mechanics are more complicated due to buckling from inplane forces and a much sooner failure under compressive stress compared to tensile stress [1]. Paper fails under compressive bending stress much sooner than under tensile conditions.

When a film is deposited on a compliant substrate, the substrate also deforms considerably, which reduces the stress in the film [5]. Considering the post-It ${ }^{\mathrm{TM}}$ paper as a compliant substrate with $\mathrm{d}_{\text {substrate }} \cong 100 \mu \mathrm{m}$ and the silver ink film to be measured around $\mathrm{d}_{\text {film }} \cong 5 \mu \mathrm{m}$ on average, then stress $\sigma_{\text {film }}$ in the metal silver film can be described by (1):

$$
\sigma_{\text {film }}=\frac{\varepsilon_{\mathrm{M}} \mathrm{Y}_{\text {film }}^{*}}{1+\left(\mathrm{Y}_{\text {film }}^{*} \mathrm{~d}_{\text {film }}\right) /\left(\mathrm{Y}_{\text {substrate }}^{*} \mathrm{~d}_{\text {substrate }}\right)} ;
$$

where $\varepsilon_{M}=\varepsilon_{0}+\left(\alpha_{\text {film }}-\alpha_{\text {substrate }}\right) \Delta \mathrm{T}$

Where $\mathrm{Y}_{\text {substrate }}^{*}$ and $\mathrm{Y}_{\text {film }}^{*}$ are respectively the biaxial elastic modulus (Young's modulus) of the paper substrate and the silver ink film. $\varepsilon_{\mathrm{M}}$ is the total mismatch strain between the substrate and the film, and $\varepsilon_{0}$ is the mismatch strain in the film, which is positive when there is an induced tensile strain. $\Delta \mathrm{T}$ is the temperature change and $\alpha_{\text {film }}-\alpha_{\text {substrate }}$ is the difference in the thermal expansion coefficients of the film and the substrate. The silver ink film is less elastic than the compliant substrate, and has a Young's modulus of around 80 $\mathrm{GPa}$. So given the elastic modulus of silver ( $\mathrm{Y}_{\mathrm{film}}^{*}=80 \mathrm{GPa}$ ) and that of our cellulose fiber paper $\left(\mathrm{Y}_{\text {substrate }}^{*}=5 \mathrm{GPa}\right)$, the relationship $Y_{\text {film }}^{*} \times d_{\text {film }}$ and $Y_{\text {substrate }}^{*} \times d_{\text {substrate }}$ becomes comparable in magnitude and value. This shows that both the film and the substrate have equal strength, which gives rise to more complicated mechanical behaviors that are not going to be addressed in this paper. This near equality arises, taking into consideration the maximum thickness of silver ink film $\mathrm{d}_{\text {film }}=5 \mu \mathrm{m}$, but still the substrate itself demonstrates a higher number. Therefore, taking into consideration that we have an even thinner metal film, in this case the stress in the silver film depends on the substrate's mechanical behavior (viz. thickness and elastic modulus), where the stress in the substrate is expressed by (2) [5]:

$\sigma_{\text {substrate }}=-\frac{\sigma_{\text {film }} \mathrm{d}_{\text {film }}}{d_{\text {substrate }}}$

Where $\sigma_{\text {substrate }}$ is the stress in the substrate and $\sigma_{\text {film }}$ is the stress in the metal film. Since our relationship exhibits a near equality, the stress in film is reduced by a factor of two from a similar film deposited on a stiffer substrate:

$\sigma_{\text {film,stiff sub }}=2 \times \sigma_{\text {film,compliant sub }}$.

When testing our sample under different bending conditions, strain is applied on the top surface of our sensors. 
1 Although strain levels will be minimal and will not lead to 2 major cracks or discontinuity, however the strain induced from 3 bending will lead to changes in the resistance of the silver ink layer and in the electrical behavior of the sensors. The resistive strain sensitivity under uniform strain distribution can be shown by [5]:

$8 r=r_{0} \frac{\left(1+\varepsilon_{1}\right)}{\left(1-v \varepsilon_{1}\right)^{2}}$

Where " $\mathrm{r}$ " is the measured resistance under bending, " $\mathrm{r} 0$ " is the initial resistance under no bending condition, " $\varepsilon_{1}$ " is the induced strain in the metal film, and " $v "$ is the Poisson ratio of the metal film $\left(v_{\mathrm{Ag}}=0.37\right)$.

Applied strain can be also calculated from the bending radius curvature and the material properties of both our substrate and sensor film. In our case, we have a stiff metal film (silver particles) on a compliant substrate (cellulose paper), thus the neutral plane surface shifts from the mid-surface and toward the film. Therefore, the strain on the top surface $\varepsilon_{\text {top }}$ is further reduced, and is expressed by (4) [5]:

$$
\varepsilon_{\text {top }}=\left(\frac{d_{\text {film }}+d_{\text {substrate }}}{2 R}\right) \frac{\left(1+2 \eta+\chi \eta^{2}\right)}{(1+\eta)(1+\chi \eta)}
$$

where $\eta=\frac{d_{\text {film }}}{d_{\text {substrate }}}$ and $\chi=\frac{Y_{\text {film }}}{Y_{\text {substrate }}}$

Where " $R$ " is the bending radius of curvature. Using this formula, and considering we have a large area blanket thin metal film, by plugging in our constants and the different bending radii used in this work, we can calculate a maximum strain $\varepsilon_{\text {top }}$ of $0.2 \%$ under a bending radius of $\mathrm{R}=1.5 \mathrm{~cm}$.

\section{Bending Effect on PAPer Sensors}

In this section, we investigate in depth each of the sensor characteristics while bent at different bending radii, $5 \mathrm{~cm}$ bending radius down to $1.5 \mathrm{~cm}$ bending radius. We intentionally limited our study to such range, since our sensory platform targets wearable applications, commonly placed on the chest (no bending - slight surface irregularity), around the arm and around the wrist, where the smallest bending radius encountered would be around the wrist joint with an average bending radius down to $2 \mathrm{~cm}$ [6]. In fact, the average wrist circumference, including newborn babies and adults, is about $14.5 \mathrm{~cm}$ [6] which approximately leads to an average bending radius at the joints of $2.3 \mathrm{~cm}$.

\section{A. Parallel-plate capacitive structure}

Considering the pressure sensor (Fig. 1g), its initia\$3 capacitance $\mathrm{C}_{\text {eff }}$ under no bending conditions is defined by (5)54

$$
\mathrm{C}_{\mathrm{eff}}=\frac{\varepsilon_{0} \varepsilon_{\mathrm{eff}} \times \mathrm{A}}{\mathrm{d}_{\mathrm{eff}}}
$$

Where " $\varepsilon_{\text {eff }}$ " is the effective relative permittivity of both aif and the cleanroom wipe, " $\varepsilon_{0}$ " is the vacuum permittivity $\left(\varepsilon_{0}=\right.$

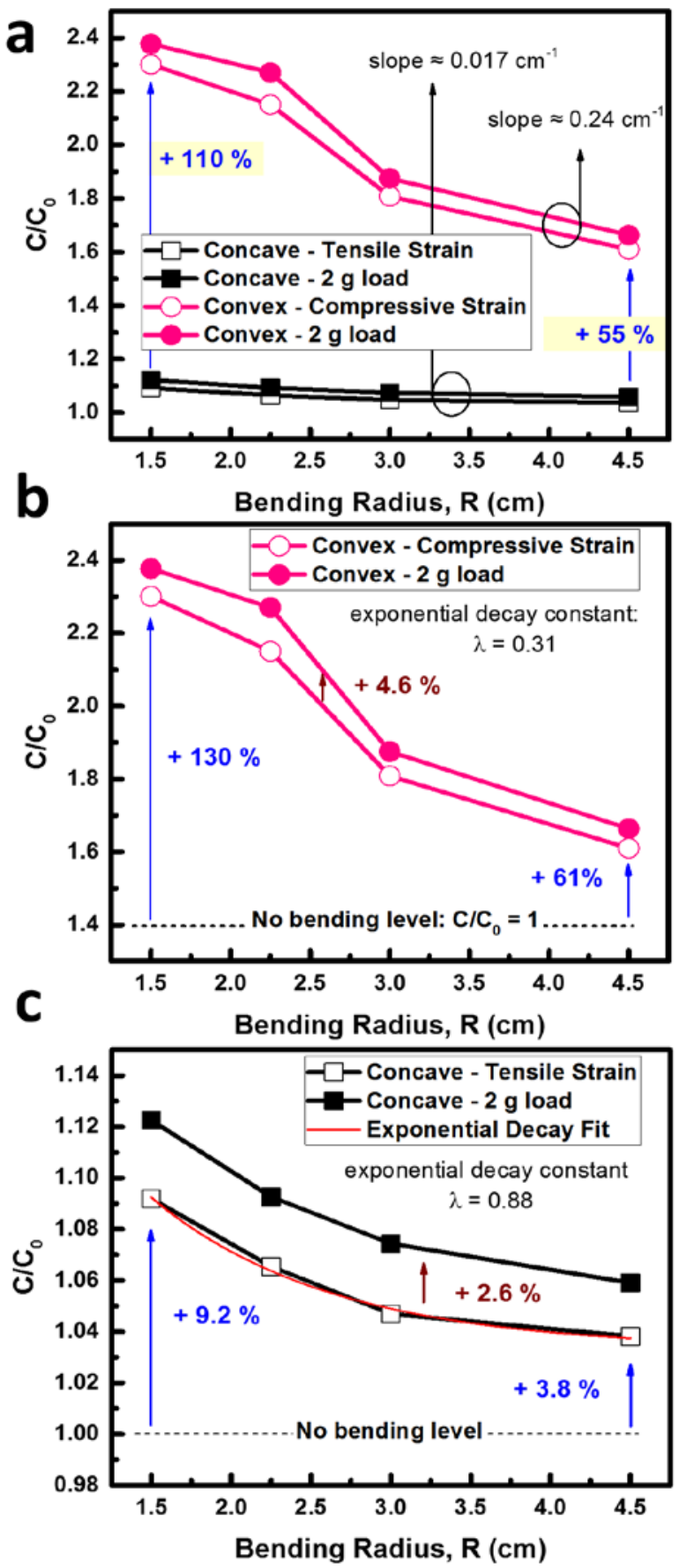

Fig. 2. (a) Plot showing the changes in the initial normalized value of the pressure sensor structure under various bending radii down to $\mathrm{R}=1.5 \mathrm{~cm}$, under loading (of $2 \mathrm{~g}$ mass) and no load conditions. (b) Compressive straininduced effect through a convex bending of the pressure sensor, and (c) Tensile strain-induced effect through a concave bending condition of the pressure structure.

$8.86 \times 10^{-12} \mathrm{~F} \cdot \mathrm{m}^{-1}$ ) and " $\mathrm{d}_{\mathrm{eff}}$ " is the total thickness of the dielectric layer (airgap and cleanroom wipe). Our measured value of capacitance may be thought of as two capacitors in series, one a perfect parallel plate capacitor filled with the dielectric cleanroom wipe material $\left(\mathrm{C}_{1}\right)$ and the other capacitor $\left(\mathrm{C}_{2}\right)$ is a perfect parallel plate capacitor filled with air [7]. Thus total device capacitance is calculated as: 

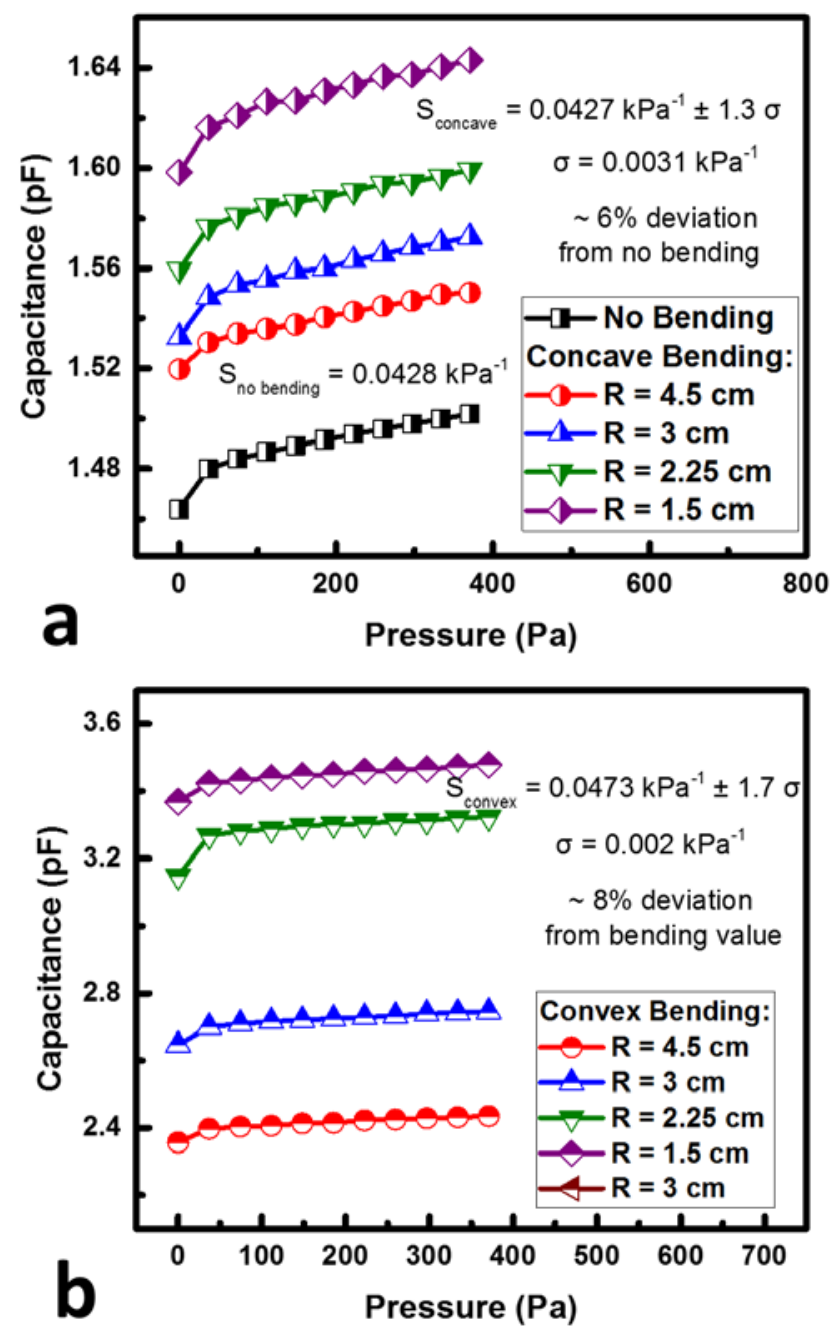

Fig. 3. Plots in (a) and (b) display a comparison of the pressure sensitivity before bending and after concave and convex bending respectively, under-various bending conditions down to $\mathrm{R}=1.5 \mathrm{~cm}$.
1

$$
\frac{1}{\mathrm{C}_{\mathrm{eff}}}=\frac{1}{\mathrm{C}_{1}}+\frac{1}{\mathrm{C}_{2}}
$$

where $\mathrm{C}_{1}=\frac{\varepsilon_{0} \varepsilon_{\text {wipe }} \times \mathrm{A}}{\mathrm{d}_{\text {wipe }}}$ and $\mathrm{C}_{2}=\frac{\varepsilon_{0} \varepsilon_{\text {air }} \times \mathrm{A}}{\mathrm{d}_{\text {air }}}$

Replacing the following parameters in (7), with $d_{\text {eff }}=690 \mu \mathrm{m}_{\mathrm{B} 2}$ $\varepsilon_{\text {wipe }}=4.09, \varepsilon_{\text {air }}=1.0005, \mathrm{~d}_{\text {wipe }}=600 \mu \mathrm{m}$ and $\mathrm{d}_{\text {air }}=90 \mu \mathrm{m}$, w6 3 get:

$$
\varepsilon_{\text {eff }}=\frac{\mathrm{d}_{\text {eff }} \times \varepsilon_{\text {wipe }} \times \varepsilon_{\text {air }}}{\left(\varepsilon_{\text {air }} \times d_{\text {wipe }}\right)+\left(\varepsilon_{\text {wipe }} \times d_{\text {air }}\right)}=2.29
$$

Under bending conditions, both concave and convex, the dielectric thickness of bent sensor $\mathrm{d}_{\mathrm{eff}}{ }^{\prime}$ is reduced due to 69 tension or compression, and airgaps are also compressed leading to an increase in the dielectric constant. Thus, $\mathrm{d}_{\mathrm{eff}}{ }^{\prime} 71$ $\mathrm{d}_{\mathrm{eff}}$ and $\varepsilon_{\mathrm{eff}}{ }^{\prime}>\varepsilon_{\mathrm{eff}}$, leading to an increase in capacitance as the $\mathrm{g}^{2}$ sensor is bent to smaller bending radii $\left(\mathrm{C}_{\text {eff }}{ }^{\prime}>\mathrm{C}_{\mathrm{eff}}\right)_{74}^{3}$
18
Moreover, an additional capacitance $\mathrm{C}_{3}$ appears in parallel with Ceff', due to stray capacitances between the two edges of the sample as they are bent towards one another. $C_{3}$ is roughly defined as the capacitance between the edges of the sample, where the dielectric is air, and the corresponding thickness $d_{2}$ is defined by the bending radius ' $R$ ' of the sample $\left(d_{2}=2 R\right)$. Since our bending radii are relatively big (in centimeter range), this stray capacitance $C_{3}$ is small since $d_{3}>>>d_{\text {eff }}$, and $\varepsilon_{3} \approx \varepsilon_{\text {air }}<<<\varepsilon_{\text {eff }}$. $C_{3}$ becomes more pronounced as bending $\mathrm{R}$ decreases and $\mathrm{d}_{3}$ becomes smaller. As a whole, combining both effects, $\mathrm{C}_{\text {total }}=\mathrm{C}_{\text {eff }}{ }^{\prime}+\mathrm{C}_{3}$, as bending radius decreases both $\mathrm{C}_{\text {eff }}$ and $\mathrm{C}_{3}$ increase, leading to an overall increase in the observed capacitance of the device $\left(\mathrm{C}_{\text {total }}>>\mathrm{C}_{\text {eff }}\right)$.

Our study also shows that our experimental data are in accordance with our theoretical expectations. We can clearly see that as the pressure sensor is bent, there is a direct increase in the initial capacitance $\mathrm{C}_{0}=1.4637 \mathrm{pF}$ (Fig. 2a). Considering the bending radius $\mathrm{R}=4.5 \mathrm{~cm}$, the convex state displays a 61 $\%$ increase of $\mathrm{C}_{0}$ from the unbent state (Fig. 2b), whereas in the concave condition, we perceive an even higher shift of $\mathrm{C}_{0}$ by $3.8 \%$ (Fig. 2c). The higher shift in convex state is explained by a higher value of $\mathrm{C}_{3}$. In fact, in the concave state, the distance $d_{3}$ is defined by the bending radius, the thickness of the paper substrate, and the thickness of the bending setup. Thus, $d_{3}$, concave $=2 R+d_{\text {paper }}+d_{\text {bending setup. In contrast, in the }}$ convex case, the metal contacts are directly facing one another and thus the dielectric of $\mathrm{C}_{3}$ is only defined by the bending radius, with $d_{3}$, convex $=2 R$. Since $d_{3}$, concave $>d_{3}$, convex,$C_{3}$, convex $>\mathrm{C}_{3}$, concave, then we expect that $\mathrm{C}_{\text {convex }}>\mathrm{C}_{\text {concave. }}$. And as we keep decreasing the bending radius down to $1.5 \mathrm{~cm}$, the capacitance value in the bent conditions keeps increasing. We then test the effect of pressure under the different bending conditions by applying a 0.2 grams of Polydimethylsiloxane (PDMS) load (1 cm diameter circular shape) on the top surface of our sensor. This PDMS load applies a compressive
54 sensitivity of the concave bending condition is determined by a slope of Slope concave $=0.017 \mathrm{~cm}^{-1}$, whereas the convex state is determined by Slope $e_{\text {convex }}=0.24 \mathrm{~cm}^{-1}$ which is about an order of magnitude higher response to change in the bending state of the sample. We believe that this greater change in the convex state can be due to a slightly denser dielectric when the cleanroom wipe is under compression, hence leading to a higher $\varepsilon_{\text {eff }}$ and higher $C_{\text {eff. }}$ In contrast, under tensile concave bending, the microfibrils are further stretched out from one 


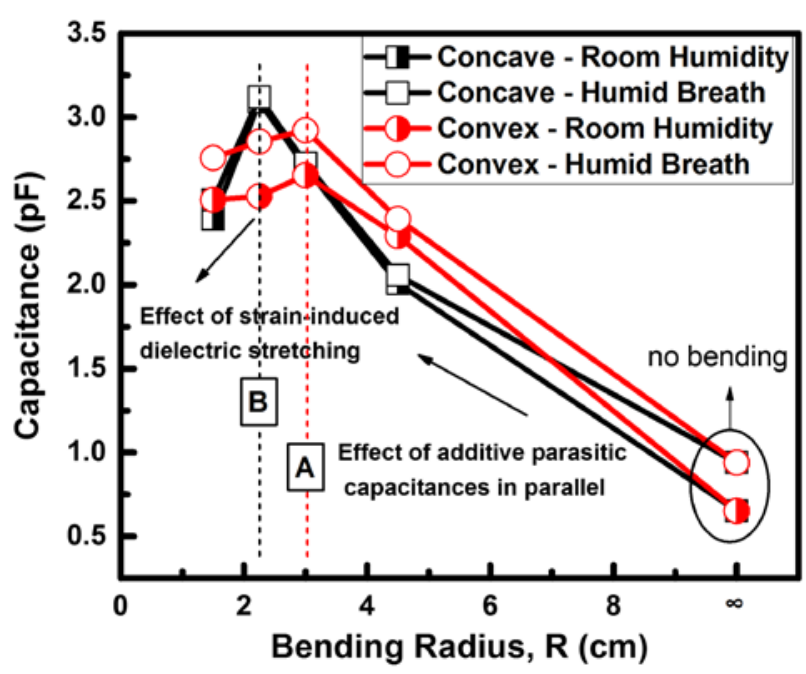

Fig. 4. Effect of concave (tensile strain) and convex (compressive strain) bending on the mechanics and electrical characteristics of the interdigitated capacitive structure of the humidity sensor. Two regimes can be distinguished8 due to an overlap between strain-induced material changes and parasitics.

another, which leads to a more porous air-filled structuri4 translated into a decrease in the dielectric constant and thus 35 smaller $\mathrm{C}_{\text {eff. }}$.

We then test the change in pressure sensitivity when thgs sensor is under these different bending conditions. The dat39 plots in Fig. 3a and b show that the bent sensors display 40 similar sensitivity trend as the non-bent sample. We exhibit 91 first linear regime up to $37 \mathrm{~Pa}$ that has a slightly highe 42 sensitivity than the second linear regime that extends up tq $370 \mathrm{~Pa}$. Note that $370 \mathrm{~Pa}$ is not our high pressure limit, but we did not need to go further for the purpose of this experiment4 Further details about the high limit of this sensor is present in our previous publication along with the analysis on the pressure sensor behavior [2]. Overall we notice on average $\mathrm{a}_{4}$ stable sensitivity to pressure. In the no bending state, the sensor has a sensitivity of $S_{\text {no bending }}=0.0428 \mathrm{kPa}^{-1} \pm 0.00128$

$\mathrm{kPa}^{-1}$, while the concave bending conditions display oñ1 average a sensitivity of $S_{\text {concave }}=0.0427 \mathrm{kPa}^{-1} \pm 0.0018 \mathrm{kPa}^{-1} .2$ Using statistical analysis, we calculate the standard deviation 3 to be $\sigma=0.0031$ and our sensitivity values in the concave conditions to be in the range of $\pm 1.3 \sigma$, with an average of $6 \% 74$ deviation from Sno bending (Fig. 3a). Similarly, for the 5 convex case, we determine an average sensitivity of $\mathrm{S}_{\text {convex }} \overline{\overline{7}} 6$ $0.0473 \mathrm{kPa}^{-1} \pm 0.0023 \mathrm{kPa}^{-1}$. The standard deviation is 77 calculated to be $\sigma=0.002$ and the convex sensitivity values to be within $\pm 1.7 \sigma$, with an average of $8 \%$ deviation from the no bending state (Fig. 3b). Overall, our numbers seem to be statistically consistent as we are still within the $3 \sigma$ region $_{81}$ Thus, we conclude that our sensor demonstrates good stability for pressure sensitivity even under a variety of tensile an 83 compressive bending conditions.

\section{B. Interdigitated electrodes structure}

The humidity sensor has an interdigitated fingers structure (Fig. 1h) which can be decomposed into $\mathrm{C}_{1}$ capacitances measured in between the lateral fingers of the structure, and $\mathrm{C}_{2}$ capacitances measured in the spacing between the vertical finger and the lateral fingers in proximity. $\mathrm{C}_{1}$ capacitances are parallel to one another, and $\mathrm{C}_{2}$ capacitances are as well parallel to one another. Thus, they their respective equivalent capacitance values can be expressed by (8):

$C_{1, \text { eq }}=C_{1}+C_{1}^{\prime}+C_{1}^{\prime \prime}+\cdots ;$ where $C_{1, \text { eq }}=\frac{\varepsilon_{0} \varepsilon_{\text {paper }} \times A}{d_{1}}$
$C_{2, \text { eq }}=C_{2}+C_{2}^{\prime}+C_{2}^{\prime \prime}+\cdots ;$ where $C_{2, \text { eq }}=\frac{\varepsilon_{0} \varepsilon_{\text {paper }} \times A}{d_{2}}$

The dielectric material of both $\mathrm{C}_{1, \text { eq }}$ and $\mathrm{C}_{2, \text { eq }}$ is the paper substrate corresponding to a dielectric constant $\varepsilon_{\text {paper }}$ " $\mathrm{A}$ " the corresponding cross-sectional area, and " $\mathrm{d}_{1}$ " and " $\mathrm{d}_{2}$ " are the distances between the capacitor's metal fingers respectively for $C_{1}$, eq and $C_{2}$, eq. Since $C_{1}$, eq and $C_{2}$, eq are in series, the equivalent capacitance of the whole sensor structure under no bending condition is expressed by (9):

$4 \frac{1}{\mathrm{C}_{\mathrm{eq}}}=\frac{1}{\mathrm{C}_{1, \mathrm{eq}}}+\frac{1}{\mathrm{C}_{2, \mathrm{eq}}}$

$5 \Rightarrow \mathrm{C}_{\mathrm{eq}}=\frac{1}{\frac{1}{\mathrm{C}_{1, \mathrm{eq}}}+\frac{1}{\mathrm{C}_{2, \mathrm{eq}}}}=\frac{\varepsilon_{0} \varepsilon_{\text {paper }} \times \mathrm{A}}{\mathrm{d}_{1}+\mathrm{d}_{2}}$

When the structure is bent in a lateral direction (along the direction of the horizontal fingers), we assume that the value of $\mathrm{C}_{1}$, eq is not affected and remains a constant, especially under the large bending radii we are demonstrating. Under this assumption, our equivalent capacitance value can be simplified to $\mathrm{C}_{\text {eq }} \cong \mathrm{C}_{2 \text {, eq }} \propto 1 / \mathrm{d}^{2}$. When the sensor is bent laterally in a concave manner, two effects occur simultaneously: (A) First, we exhibit the presence of stray capacitances $C^{*}$ in parallel to $C_{2}$, eq, mainly due to the approach of the metal fingers and pads to one another as we fold the structure closer together from each end. This effect is usually very pronounced as the sensor size gets bigger, which is the case in our current work (cm dimensions). In this case, $\mathrm{C}^{*}$ is defined by the dielectric constant of air due to bending curvature and paper:

$C^{*}=\frac{\varepsilon_{0} \times \varepsilon^{*} \times A}{\left(d_{\text {air }}+d_{\text {paper }}\right)}=\frac{\varepsilon_{0} \times \varepsilon^{*} \times A}{\left(2 R+d_{\text {paper }}\right)}$

$$
\text { where } \varepsilon^{*}=\frac{d^{*} \times \varepsilon_{\text {paper }} \times \varepsilon_{\text {air }}}{\left(\varepsilon_{\text {air }} \times d_{\text {paper }}\right)+\left(\varepsilon_{\text {paper }} \times d_{\text {air }}\right)}
$$

Where $\varepsilon^{*}$ is the effective dielectric constant under bending condition, $\varepsilon_{\text {paper }}$ and $\varepsilon_{\text {air }}$ are respectively the dielectric constants of paper and air, and $\mathrm{d}^{*}=\left(\mathrm{d}_{\mathrm{air}}+\mathrm{d}_{\mathrm{paper}}\right)$.

(B) Second, we exhibit strain-induced stretching of the cellulose fibers of the paper, leading to a change in the dielectric constant of paper. This effect mostly affects the $C_{2}$, eq capacitance due to the bending direction, and thus we expect to see significant changes in $\mathrm{C}_{2}$, eq value as the sample is bent at smaller radii. As mentioned, the two effects described above 


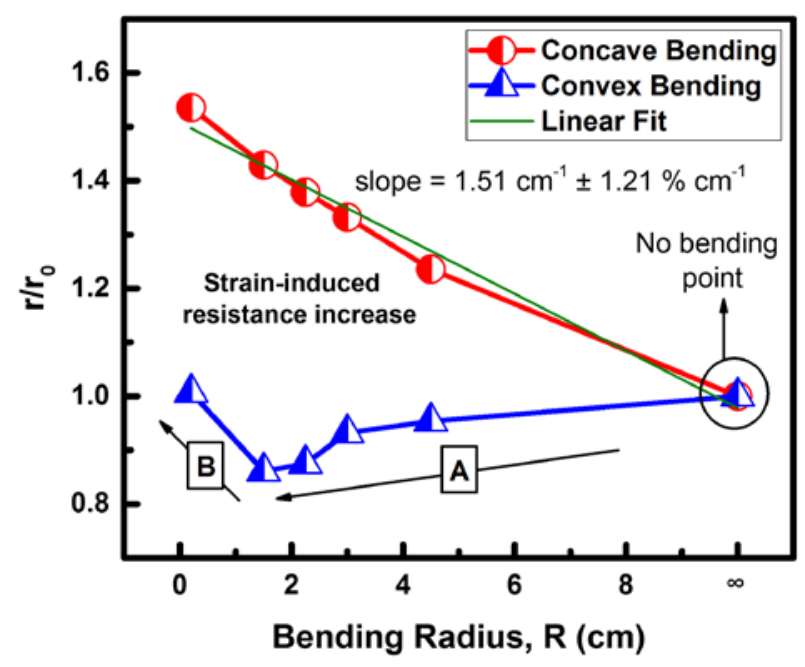

Fig. 5. Effect of concave and convex bending conditions on the electrical ${ }_{-}^{5} 3$ characteristics and resistive mechanical structure of the temperature sensor. ${ }^{34}$ Two regimes A and B can be distinguished only for the compressive strain̄5 case due to complex structural changes happening in the silver ink. occur simultaneously, however for simplicity, either one o $\$ 8$ them is seen to be separately more dominating in a certai ${ }^{9}$ bending range, and thus assumptions can be made to attribut 80 changes in the final capacitance values to one specific behavior. Thus, under bending conditions, the total 1 capacitance $\mathrm{C}_{\mathrm{T}}$ is represented by (11):

$\mathrm{C}_{\mathrm{T}}=\mathrm{C}_{2, \mathrm{eq}}+\mathrm{C}^{*}$

Assuming $C_{1}$ negligible under lateral bending, as we first start 66 bending the sensor structure, the applied strain is not enough to stretch prominently the cellulose fibers, thus the effect of 69 stretching is negligible and $\mathrm{C}_{2}$, eq is considered to stay constant In this case, the effect of $\mathrm{C}^{*}$ becomes dominant, and as bending radius " $\mathrm{R}$ " decreases, distance " $\mathrm{d}_{\text {air }}$ " expressed in $(\mathbf{1 0} 1$ decreases, leading to an overall increase in $\mathrm{C}^{*}$ and in the tota 2 measured capacitance $\mathrm{C}_{\mathrm{T}}$ of the sensor. As bending radius is further reduced, the effect of stretching on $\mathrm{C}_{2 \text {, eq }}$ dominates the effect on the final capacitance. As mentioned above, higher 76 applied strains will lead to stretching of the cellulose fibers, 76 making the structure of the paper less tightly packed. This 8 effect leads to a noticeable decrease in the dielectric constant 9 of $\mathrm{C}_{2}$, eq, more prominent that the small increase occurring on $\mathrm{C}^{*}$. Therefore, taking $\mathrm{C}^{*}$ as a constant at smaller bending radid $\mathrm{C}_{2}$, eq decreases and leads to an overall decrease in the total 82 capacitance $\mathrm{C}_{\mathrm{T}}$ of the structure.

Our experimental data in Fig. 4 is in accordance with our 83 expected results. We test the response of the humidity sensor 85 to different bending conditions and under two different relative humidity levels: (1) at room temperature ( $\sim 6 \% \mathrm{RH}_{87}^{6}$ and (2) applying humid breath $(\sim 76 \% \mathrm{RH})$. Clearly, at higher 88 humidity levels, we will see slightly higher capacitance values 88 due to an increase in the dielectric constant [2]. This effect is translated in Figure 4 by an increase of $44 \%$ in the $C_{0}$ valug ${ }_{1}$ under no bending. The effect of bending is seen to be the samg for the samples exposed to either room humidity or humid breath. With a closer look at the room humidity case, we see that under concave tensile bending there is a 5 times increase in the capacitance value as bending radius decreases down to $\mathrm{R}=2.25 \mathrm{~cm}$. Beyond this point, we witness a $23 \%$ decrease in the capacitance value. Under convex compressive bending, we initially see a 4 times increase in $\mathrm{C}_{\text {no bending as bending radius }}$ goes down to $\mathrm{R}=3 \mathrm{~cm}$. Then, we observe again a $6 \%$ decrease in the capacitance value. Moreover, for the convex case, the shift in capacitance between room humidity and humid breath has decreased down to $9 \%$ on average throughout the bending modifications, whereas in the concave case this shift has been decreased down to about 2\% (Fig. 4). This shows that under bending conditions, we witness a decrease in the sensitivity of our humidity sensors, which can be explained by a smaller contact area between the exerted humid stimuli and the surface of the sensor. We believe that this is a setup related variation, where the surface of the sensor was not fully exposed to the humid breath. This is seen more prominently in the concave case since a larger portion of the structure is bent downwards from either side, and in our experimental setup, humid breath was exerted solely from the top surface in a perpendicular fashion.

\section{Resistive Configuration}

Lastly for the resistive temperature sensor (Fig. 1i), the bending mechanics are much simpler. We test the variations in the readout of the sensor under both concave and convex bending, down to a $2 \mathrm{~mm}$ bending radius (Fig. 5). Under tensile concave conditions, the metal undergoes strain $(\varepsilon=$ $\Delta \mathrm{L} / \mathrm{L}$ ) and experiences a resistance change $r_{\varepsilon}$ expressed by (12) [8]:

$r_{\varepsilon}=r_{0} \times(1+\mathrm{GF} \times \varepsilon)$

Where the strain sensitivity or gage factor (GF) is a dimensionless quantity, " $\mathrm{r}$ " is the initial resistance of the sensor under no strain, " " is the length of the flat sample and " $\Delta \mathrm{L}$ " the change in the length induced by an applied strain $\varepsilon$. As the sample is bent to smaller bending radii, it will be stretched in length due to higher strain levels. As a result, we expect an increase in the total resistance of the structure.

This behavior is clearly seen in Fig. 5 where we witness a linear relationship between resistance and bending radius under tensile bending conditions. As bending radius is decreased and strain levels increase, resistance values linearly increase with a sensitivity of $15.1 \mathrm{~mm}^{-1}$. Under convex bending, the situation is a bit different and we can distinguish two different regions (A) and (B) (Fig. 5). In region (A), we first witness a decrease in resistance as we bend the sample down to $1.5 \mathrm{~cm}$ bending radius. As the metal film is compressed under compressive bending, the silver composites are brought closer together and overlap, leading to improved packing density which is highlighted by enhanced conductivity and a decrease in resistance. However, as we compress further the sample down to $2 \mathrm{~mm}$ bending radius, we enter region (B) where we experience again an increase in 
1 resistance. Once the Ag particles overlap, the top layer of the 2 metal film is experiencing compression, but the bottom part is 3 experiencing tensile strain which leads to the increase in 4 resistance seen in the plot of Fig. 5.

We then test the effect of bending on the sensitivity of the sensor. In our experimental setup, temperature is applied to the bottom of the sample through a thermal chuck, thus we expect very minimal thermal loss through the porous insulating paper substrate.

For this reason, we test the offset between the real applied temperature and the temperature seen by the sensor (Fig. 6a). "Seen" temperature is measured through a thermocouple placed on the top metal layer of the sensor. The plot in Fig. 6a shows a linear relationship where the change in "seen" temperature is expressed with respect to the change in "real" temperature by $\Delta \mathrm{T}_{\text {seen }}=0.89 \pm 0.001 \Delta \mathrm{T}_{\text {real }}$ and $\mathrm{T}_{\text {seen }}=0.89+$ $2.21 \mathrm{~T}_{\text {real }}\left[{ }^{\circ} \mathrm{C}\right]$. Then, we test our sensor's sensitivity to temperature changes under no bending and under both concave and convex bending conditions, with a fixed bending radius of $\mathrm{R}=3 \mathrm{~cm}$. As seen in Fig. $\mathbf{6 b}$, the non-bent sample displays an expected linear behavior with a sensitivity of Snot bent $=0.0115 \Omega .^{0} \mathrm{C}^{-1}$. The corresponding temperature coefficient of resistance TCR " $\alpha$ " is expressed by:

$\alpha=\frac{\Delta r}{\Delta T} / r_{0}$

Where " $\alpha$ " is expressed in ${ }^{\circ} \mathrm{C}^{-1}$. For this case, the TCR is calculated to be $\alpha_{\text {not bent }}=4.1 \times 10-3{ }^{\circ} \mathrm{C}^{-1} \pm 4.4 \times 10^{-5}{ }^{\circ} \mathrm{C}^{-1}$, while the theoretical value of TCR for the silver metal is $\alpha_{\mathrm{Ag}}=3.8 \times$ $10^{-3}{ }^{\circ} \mathrm{C}^{-1}$. This highlights the high quality of our metal film, with no defects affecting its thermal or electrical conductivity. Still, when the sample is bent, we again witness a perfect linear behavior in both bending cases with sensitivities of $\mathrm{S}_{\text {concave }}=0.0116 \Omega .^{\circ} \mathrm{C}^{-1}$ and $\mathrm{S}_{\text {convex }}=0.0113 \Omega .{ }^{\circ} \mathrm{C}^{-1}$, respectively for concave and convex bending conditions, with only up to $1.9 \%$ deviation from the no bending state. On average, we determine a sensitivity of $S_{\text {Avg }}=0.0115 \Omega .^{\circ} \mathrm{C}^{-1}{ }^{ \pm} 5$ $4.4 \times 1.14 \sigma$, where the standard deviation is calculated to be $\$ 6$ $=1.37 \times 10^{-4}$. The corresponding average TCR is also evaluate 7 to be $\alpha_{\text {avg }}=3.86 \times 10^{-3}{ }^{\circ} \mathrm{C}^{-1} \pm 5.37 \times 10^{-5}{ }^{\circ} \mathrm{C}^{-1}$, again displayin 58 the high performance and quality of our sensor even under 9 bending conditions.

\section{ConClusion}

In this paper the essential mechanics of cellulose paper substrate based electronics and sensors fabricated on paper are 66 described and discussed. Compared to alternative techniques of making flexible sensors using polymers or functionalized solution-processed inks, using off-the-shelf papers has a much lower-cost, simpler process and definitely guaranteeing more 6 environmental friendly "green" electronics. Through this stud 69 on the stress-strain mechanics of paper electronics, pape 1 presents an advantage for wearable electronics due to it\$2 stretchy fibrous structure. We also infer that, electronics bui 3 on paper will see very minimal strain and stress, henc 75
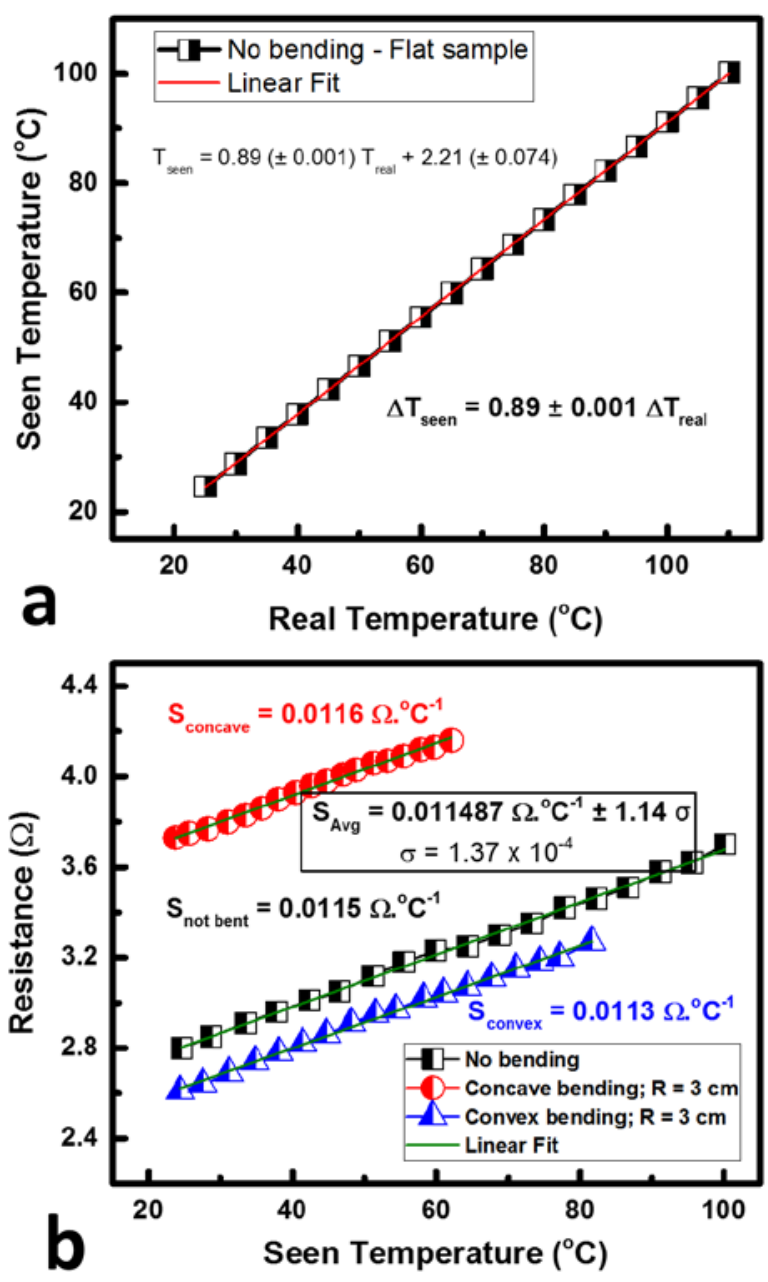

Fig. 6. (a) Plot displaying the linear relationship between the external temperatures applied during the experiment from the bottom of the sensor and the actual temperature seen by then metal film of the sensor. (b) Sensitivity plots comparing the temperature sensitivity coefficient before and after bending conditions (both concave and convex) with a fixed bending radius of $\mathrm{R}=3 \mathrm{~cm}$.

ensuring stability of performance under tensile and compressive bending conditions. Reliability of the metal lines after multiple cycles of bending on cellulose paper can be referred to in the following study by Jennifer Lewis [3], ensuring reliability and stability of paper electronics after deformation. In that aspect, the presented study provides necessary information everyone needs to know when developing paper-based electronics and systems. Our paper sensors exhibit good stability in terms of sensitivity and performance while bent at various bending radii, highlighting their potential use in affordable healthcare monitoring applications and IoE applications worn on the body or placed on any asymmetric architecture existing in nature.

\section{REFERENCES}

[1] P.-J. Gustafsson and K. Niskanen, "Paper as an engineering material," in Mechanics of Paper Products, K. Niskanen, Ed., ed: Walter de Gruyter, 2012, pp. 5-22.

[2] J. M. Nassar, M. D. Cordero, A. T. Kutbee, M. A. Karimi, G. A. T. Sevilla, A. M. Hussain, A. Shamim, and M. M. Hussain, "Paper Skin Multisensory Platform for Simultaneous Environmental Monitoring," Adv. Mater. Technol., vol. 1, p. 1600004, 2016. 
[3] A. Russo, B. Y. Ahn, J. J. Adams, E. B. Duoss, J. T. Bernhard, and J. A. Lewis, "Pen-on-paper flexible electronics," Adv. Mater., vol. 23, pp. 3426-3430, 2011.

[4] D. Thibaud, "Mechanical properties of materials made of nanocellulose," Master's degree Solid Mechanics, KTH School of Engineering Sciences, 2011.

[5] S. Wagner, H. Gleskova, I.-C. Cheng, J. C. Sturm, and Z. Suo, "Mechanics of TFT technology on flexible substrates," in Flexible Flat Panel Displays. vol. 14, ed, 2005, pp. 263-83.

[6] "Wrist Measurement Chart", Naturesbabyblankets.com. [Online]. Available: http://www.naturesbabyblankets.com/page/1263812. [Accessed: 06- Nov- 2016].

[7] T. T. Grove, M. F. Masters, and R. E. Miers, "Determining dielectric constants using a parallel plate capacitor," Am. J. Phys., vol. 73, pp. 5256, 2005.

[8] W. C. Young and R. G. Budynas, Roark's formulas for stress and strain vol. 7: McGraw-Hill New York, 2002.

Joanna M. Nassar received her B.S. in Physics in 2012 from the American University of Beirut (AUB) in Lebanon, and her M.S in Electrical Engineering in 2014 from King Abdullah University of Science and Technology (KAUST) in Saudi Arabia, where she is currently pursuing her Ph.D. focusing on using disruptive affordable materials for developing flexible and stretchable skin-like sensory systems.

Muhammad M. Hussain (M'07-SM'10) received the Ph.D. degree from The University of Texas at Austin, Austin, TX, USA, in 2005. He is currently an Associate Professor with the King Abdullah University of Science and Technology, Thuwal, Saudi Arabia. He has authored 240+ research papers and holds 50+ U.S. patents/applications. Dr. Hussain is an IEEE Electron Devices Society Distinguished Lecturer and a Fellow of APS. 